\title{
Geometrical string and spin systems
}

\author{
G.K. Savvidy \\ Physics Department, University of Crete \\ 71409 Iraklion, Crete, Greece \\ and \\ Institut für Theoretische Physik \\ D-6000 Frankfurt am Main 11,Germany \\ F.J.Wegner \\ Institut für Theoretische Physik \\ Ruprecht-Karls-Universität Heidelberg \\ Philosophenweg 19, D-69120 Heidelberg, Germany
}

\begin{abstract}
We formulate the geometrical string which has been proposed in the articles [1, 2, 3] on the euclidean lattice. It is possible to find such spin systems with local interaction which reproduce the same surface dynamics. In the three-dimensional case this spin system is a usual Ising ferromagnet with additional diagonal antiferromagnetic interaction and with specially adjusted coupling constants. In the four-dimensional case the spin system coincides with the gauge Ising system [4] with an additional double-plaquette interaction and also with specially tuned coupling constants. We extend this construction to random walks and random hypersurfaces (membrane and p-branes) of high dimensionality. We compare these spin systems with the eight-vertex model and BNNNI models.
\end{abstract}




\section{Introduction}

In the articles [1, 2, 3] the authors suggest a new geometrical string, which can be considered as a natural extension of the Feynman integral over paths to an integral over surfaces in the sense, that both amplitudes coincide in the cases, when the surface degenerates into a single particle world line. This geometrical string has been formulated in continuum euclidean space $R^{d}$, where the Feynman integral coincides with the partition function for the randomly fluctuating surfaces.

In this article we formulate this surface model on a euclidean lattice, where the surface is associated with the collection of plaquettes, and we find statistical systems with local interaction, which reproduce the same surface dynamics.

In continuum euclidean space $R^{d}$ the random surface is associated with a connected polyhedral surface $M$ with vertex coordinates $X_{i}$, where $i=1, . .,|M|$ and $|M|$ is the number of the vertices. The energy functional for the polyhedral surface is defined as [1]

$$
A(M)=\sum_{<i, j>}\left|X_{i}-X_{j}\right| \cdot \Theta\left(\alpha_{i, j}\right),
$$

where

$$
\begin{gathered}
\Theta(\pi)=0, \\
\Theta(2 \pi-\alpha)=\Theta(\alpha), \\
\Theta(\alpha) \geq 0,
\end{gathered}
$$

the summation is over all edges $<i, j>$ of $M$ and $\alpha_{i, j}$ is the angle between the two neighbor faces (flat polygons) of $M$ in $R^{d}$ having a common edge $\langle i, j\rangle$.

For triangulated surfaces one can define the partition function

$$
Z(\beta)=\sum_{M \in\{M\}} \int e^{-\beta A(M)} \prod_{i \in M} d X_{i},
$$

where $\{M\}$ denotes a set of triangulations. The physical properties of the model have been discussed in [1, 2, 3].

In the present article we formulate this string on a euclidean lattice and find a corresponding lattice field theory to which it is equivalent. A priory it is not evident that an equivalent field theory exists. Particularly we will show, that for euclidean lattice surfaces, built from plaquettes, there exists a spin system whose interface energy coincides with the expression (1).

This will be done in two steps. In sect. 2 we will formulate the lattice version of the string proposed in [1, 2, 3], and in sect. 3 we will construct an appropriate spin system with local interaction. The general formulation for $d-n$-dimensional hypersurfaces in $d$-dimensional space is given in sect. 4 


\section{Surfaces on a lattice}

On the lattice a closed surface can be considered as a collection of plaquettes, whose edges are glued together pairwise. There are two essentially distinct cases. In the first case the surface is considered as a connected, orientable surface with given topology and it is assumed, that self-intersections of the surface do not produce any additional energy. The surface should be allowed to be freely intersected. In this non-self-avoiding case the definition of the surface energy, as it is defined by the expression (1), is complete.

In the second case it is assumed, that self-intersections of the surface produce an additional energy and one should define nontrivial weights associated with these intersections [3]. This "fermionic" case corresponds to an effective soft-self-avoidance of the surface, and the corresponding weights should be defined as [3]

$$
\sum_{\text {common edges }}\left|X_{i}-X_{j}\right| \cdot\left(\Theta\left(\alpha_{i, j}^{(1)}\right)+\ldots+\Theta\left(\alpha_{i, j}^{(r(2 r-1))}\right)\right),
$$

where $\alpha_{i, j}^{(1)}, . ., \alpha_{i, j}^{(r(2 r-1))}$ are the angles between the pairs of plaquettes of $M$ at the common edge $\langle i, j\rangle$ in $R^{d}$ in which $2 r$ plaquettes intersect. The order of selfintersection is $r=0,1, . ., d-1$.

On the lattice the lengths of the elementary edges $\left|X_{i}-X_{j}\right|$ are equal to the lattice constant $a$ and the angles between plaquettes are either $0, \pi / 2$ or $\pi$. Therefore we should only define quantities $\Theta(0), \Theta(\pi / 2)$ and $\Theta(\pi)$ in $(1)$, where the last one is actually equal to zero (2). In the case of infinitely heavy quarks, that is $\Theta(0)=\infty$ [2, 3], one can neglect fluctuations of the surface with overhanging plaquettes, that is with folds $\left(\alpha_{i, j}=0\right)$. Therefore only $\Theta(\pi / 2)$ remains as a free parameter of the theory.

Thus we have a full set of rules which allow to compute the energy functional and to define the surface dynamics on the lattice. These rules are: i) if two plaquettes of the closed surface intersect under the right angle, the contribution to the energy is equal to $a \cdot \Theta(\pi / 2)$, ii) if they are parallel, the contribution is equal to zero $a \cdot \Theta(\pi)=0$ and iii) if four or more plaquettes intersect on a given edge, then $a \cdot \Theta(\pi)$ has to be multiplied by the number of pairs of plaquettes which meet under a right angle. Finally the full energy associated with the lattice surface can always be written as

$$
H=\sum_{\text {over all edges }} H_{\text {edge }}
$$

where $H_{\text {edge }}$ is the energy associated with a given edge. This completely defines the surface dynamics on the lattice.

In the following we will set $a=1$ and in many cases we will suppress the coupling $\Theta(\pi / 2)$. 


\section{Equivalent spin model}

Now let us attach variables $U_{P}$ to each plaquette $P$ of the lattice [4]. By definition its value is -1 , if a given plaquette belongs to the surface $M$ and it is equal to +1 otherwise,

$$
U_{P}=-1
$$

if $P \in M$ and

$$
U_{P}=+1 \text {. }
$$

if $P \notin M$. Since only an even number of plaquettes can meet at a given lattice edge, the total product of corresponding plaquette variables $U_{P}$ surrounding an edge is always equal to one,

$$
U_{1} U_{-1} U_{2} U_{-2}=1
$$

if $d=3$ and

$$
U_{1} U_{-1} U_{2} U_{-2} U_{3} U_{-3}=1
$$

if $d=4$, where $U_{P}$ denotes corresponding plaquette variables and we choose the given edge in the 3 -rd direction when $d=3$ and in the 4 -th direction when $d=4$.

For our set of rules the energy of the lattice surface can be reexpressed through the plaquette variables $U_{P}$. The energies of all possible configurations at a given lattice edge is given by (we assume $a=1$ )

$$
H_{\text {edge }}=\frac{1}{4}\left(2-U_{1}-U_{-1}\right)\left(2-U_{2}-U_{-2}\right) \Theta(\pi / 2),
$$

if $d=3$ and by

$$
\begin{aligned}
& =\frac{1}{4}\left(2-U_{1}-U_{-1}\right)\left(2-U_{2}-U_{-2}\right) \Theta(\pi / 2) \\
& +\frac{1}{4}\left(2-U_{2}-U_{-2}\right)\left(2-U_{3}-U_{-3}\right) \Theta(\pi / 2) \\
& +\frac{1}{4}\left(2-U_{1}-U_{-1}\right)\left(2-U_{3}-U_{-3}\right) \Theta(\pi / 2),
\end{aligned}
$$

if $d=4$.

In three- and four-dimensions these formulae together with (5), define the full Hamiltonian of the desired system. As it follows from constraints (7), the local plaquette variables $U_{P}$ are not completely independent. To resolve constraints and

to describe the system in terms of independent local variables we should introduce Ising spins on the dual lattice [4].

In three dimensions, $R^{3}$, one should attach spin variables to vertices of the dual lattice and represent plaquette variables $U_{P}(7 \mathrm{a})$ in the form

$$
U_{1}=\sigma_{1} \sigma_{2}, U_{-1}=\sigma_{-1} \sigma_{-2}, U_{2}=\sigma_{1} \sigma_{-2}, U_{-2}=\sigma_{-1} \sigma_{2},
$$

where $\sigma_{r}= \pm 1$ denotes four Ising spin variables surrounding a given edge. This representation resolves the constraints (7a) and yields 


$$
H_{\text {edge }}=1-\frac{1}{2}\left(\sigma_{1} \sigma_{2}+\sigma_{2} \sigma_{-1}+\sigma_{-1} \sigma_{-2}+\sigma_{-2} \sigma_{1}\right)+\frac{1}{2}\left(\sigma_{1} \sigma_{-1}+\sigma_{2} \sigma_{-2}\right) .
$$

The full Hamiltonian is the sum of $H_{\text {edge }}$ over all elementary lattice edges (5)

$$
H=-2 J \sum_{\vec{r}, \vec{\alpha}} \sigma_{\vec{r}} \sigma_{\vec{r}+\vec{\alpha}}+\frac{1}{2} J \sum_{\vec{r}, \vec{\alpha}, \vec{\beta}}\left(\sigma_{\vec{r}} \sigma_{\vec{r}+\vec{\alpha}+\vec{\beta}}+\sigma_{\vec{r}+\vec{\alpha}} \sigma_{\vec{r}+\vec{\beta}}\right)
$$

The vector $\vec{\alpha}$ runs over the $d$ unit vectors parallel to the $d$ axes. Similarly the sum over $\vec{\alpha}$ and $\vec{\beta}$ runs over different pairs of such vectors. The Hamiltonian represents a magnetic system with competing interaction and specially adjusted coupling constants

$$
J_{\text {ferromagnet }}=4 J_{\text {antiferromagnet }} \equiv 2 \Theta(\pi / 2)
$$

Hamiltonian (11) coincides with usual Ising ferromagnet with additional diagonal antiferromagnetic interaction. As it is easy to see from (11), here the ground state is more degenerate than in the usual Ising case.

In order to resolve the constraints (7b) in four dimensions, one should attach independent spin variables $\sigma(r)$ to links of the dual lattice (or what is equivalent at the center of plaquettes of the initial lattice) [4. This allows to represent plaquette variables $(7 \mathrm{~b})$ in the form of product of spins around the dual plaquette

$$
\begin{aligned}
& U_{1}=\sigma_{2}(r) \sigma_{3}(r) \sigma_{2}\left(r-e_{3}\right) \sigma_{3}\left(r-e_{2}\right), \\
U_{-1}= & \sigma_{2}\left(r-e_{1}\right) \sigma_{3}\left(r-e_{1}\right) \sigma_{2}\left(r-e_{3}-e_{1}\right) \sigma_{3}\left(r-e_{2}-e_{1}\right), \\
& U_{2}=\sigma_{1}(r) \sigma_{3}(r) \sigma_{1}\left(r-e_{3}\right) \sigma_{3}\left(r-e_{1}\right), \\
U_{-2}= & \sigma_{1}\left(r-e_{2}\right) \sigma_{3}\left(r-e_{2}\right) \sigma_{1}\left(r-e_{3}-e_{2}\right) \sigma_{3}\left(r-e_{1}-e_{2}\right), \\
& U_{3}=\sigma_{1}(r) \sigma_{2}(r) \sigma_{1}\left(r-e_{2}\right) \sigma_{2}\left(r-e_{1}\right), \\
U_{-3}= & \sigma_{1}\left(r-e_{3}\right) \sigma_{2}\left(r-e_{3}\right) \sigma_{1}\left(r-e_{2}-e_{3}\right) \sigma_{2}\left(r-e_{1}-e_{3}\right),
\end{aligned}
$$

Together with $(8 \mathrm{~b})$ this yields

$$
H_{\text {edge }}=3-\sum_{6 \text { plaquettes }}(\sigma \sigma \sigma \sigma)+\frac{1}{4} \sum_{12 \text { right angle plaquettes }}\left(\sigma \sigma \sigma \sigma_{\alpha}\right)^{r t}\left(\sigma_{\alpha} \sigma \sigma \sigma\right) .
$$

The full gauge invariant Hamiltonian in four dimensions reads

$$
H=-\frac{4}{g^{2}} \sum_{\text {plaquettes }}(\sigma \sigma \sigma \sigma)+\frac{1}{4 g^{2}} \cdot \sum_{\text {right angle plaquettes }}\left(\sigma \sigma \sigma \sigma_{\alpha}\right)^{r t}\left(\sigma_{\alpha} \sigma \sigma \sigma\right),
$$

where $g^{2}$ is the gauge coupling constant and

$$
J_{\text {plaquettes }}=16 \cdot J_{r t \text { plaquettes }}=\frac{4}{g^{2}} .
$$

Now one can define the partition function as 


$$
Z(\beta)=\sum_{\left\{\sigma_{\vec{r}}\right\}} e^{-\beta H}
$$

Thus we have obtained the equivalent Ising models in three and four dimensions.

\section{Model in higher dimensions}

Equivalent spin systems can also be introduced in high dimensions. For this purpose we introduce an appropriate condense notation. It is also possible to extend this construction to random walks and random hypersurfaces of high dimensionality (membranes and p-branes), when the energy functional has the same nature (1),(4).

The points of the lattice are given by a $d$ - dimensional vector $\vec{r}$ whose components $r_{1}, . ., r_{d}$ are integer. In this lattice, $d-n$ dimensional elementary hyperplaquettes $\Omega_{\alpha_{1} \ldots \alpha_{n}}(\vec{r})$, (all $\alpha_{i}$ are different) are defined by $x_{\alpha_{i}}=r_{\alpha_{i}}$ and $r_{\alpha} \leq x_{\alpha} \leq r_{\alpha}+1$ for all other $\alpha$. The $d-n-1$ dimensional boundaries of the hyperplaquette $\Omega_{\alpha_{1} \ldots \alpha_{n}}(\vec{r})$ are the $2(d-n)$ hyperedges $\Omega_{\alpha_{1} \ldots \alpha_{n} \beta}(\vec{r})$ and $\Omega_{\alpha_{1} \ldots \alpha_{n} \beta}\left(\vec{r}+\vec{e}_{\beta}\right)$, where $\beta$ differs from the $\alpha_{i}^{\prime} s$ and $\vec{e}_{\beta}$ is the unit vector in $x_{\beta}$ direction.

A $d-n$ dimensional hypersurface $M_{d-n}$ in this lattice consists of a collection of the elementary hyperplaquettes $\Omega_{\alpha_{1} \ldots \alpha_{n}}(\vec{r})$. As before we introduce a variable $U_{\alpha_{1} \ldots \alpha_{n}}(\vec{r})$, which assumes the value -1 if the hyperplaquette belongs to the hypersurface $M_{d-n}$ and +1 if it does not. The hypersurface $M_{d-n}$ is closed, if each $d-n-1$ dimensional hyperedge $\Omega_{\alpha_{1} \ldots \alpha_{n+1}}(\vec{r})$ belongs totally to an even number of $d-n$ dimensional hyperplaquettes of hypersurface $M_{d-n}$.

Now we can introduce an energy of the closed $d-n$ dimensional hypersurface. This energy is a sum over all $d-n-1$ dimensional hyperedges

$$
H=\sum H_{\alpha_{1} \ldots \alpha_{n+1}}(\vec{r}) \text {. }
$$

The contribution of such a hyperedge depends on the $U$ 's of the surrounding $d-n$ dimensional hyperplaquettes. The energy can always be expressed by a polynomial in U's due to the identity

$$
H(\{U\})=\sum_{U^{\prime}} \prod_{i} \frac{1+U_{i} U_{i}^{\prime}}{2} H\left(\left\{U^{\prime}\right\}\right) .
$$

If the energy is the area of the hypersurface $M_{d-n}$, that is the number of $d-n$ dimensional elementary hyperplaquetts, then we can write it as [⿴囗口 ]

$$
H^{\text {area }}=\sum \frac{1-U_{\alpha_{1} \ldots \alpha_{n}}(\vec{r})}{2} .
$$

This is the type of Hamiltonians considered by Wegner [4]. Since each $d-n$ dimensional hyperplaquette belongs to the $2(d-n)$ hyperedges $\Omega_{\alpha_{1} \ldots \alpha_{n} \beta}(\vec{r})$ and $\Omega_{\alpha_{1} \ldots \alpha_{n} \beta}\left(\vec{r}+\vec{e}_{\beta}\right)$, we may write the contribution of $\Omega_{\alpha_{1} \ldots \alpha_{n+1}}(\vec{r})$ in the area case as

$$
H_{\alpha_{1} \ldots \alpha_{n+1}}^{a r e a}(\vec{r})=
$$




$$
\frac{1}{4(d-n)} \sum_{k}\left(2-U_{\alpha_{1} \ldots \alpha_{k-1} \alpha_{k+1} \ldots \alpha_{n+1}}(\vec{r})-U_{\alpha_{1} \ldots \alpha_{k-1} \alpha_{k+1} \ldots \alpha_{n+1}}\left(\vec{r}-\vec{e}_{\alpha_{k}}\right)\right) .
$$

Instead of that we wish to introduce an energy which counts at each hyperedge $\Omega_{\alpha_{1} \ldots \alpha_{n+1}}(\vec{r})$ the number of pairs of hyperplaquettes $\Omega_{\alpha_{1} \ldots \alpha_{k-1} \alpha_{k+1} \ldots \alpha_{n+1}}$ that meet under a right angle. Apparently this is given by

$$
\begin{gathered}
H_{\alpha_{1} \ldots \alpha_{n+1}}(\vec{r})= \\
\frac{1}{4} \sum_{i<k}\left(2-U_{\alpha_{1} \ldots \alpha_{i-1} \alpha_{i+1} \ldots \alpha_{n+1}}(\vec{r})-U_{\alpha_{1} \ldots \alpha_{i-1} \alpha_{i+1} \ldots \alpha_{n+1}}\left(\vec{r}-\vec{e}_{\alpha_{i}}\right)\right) \\
\left(2-U_{\alpha_{1} \ldots \alpha_{k-1} \alpha_{k+1} \ldots \alpha_{n+1}}(\vec{r})-U_{\alpha_{1} \ldots \alpha_{k-1} \alpha_{k+1} \ldots \alpha_{n+1}}\left(\vec{r}-\vec{e}_{\alpha_{k}}\right)\right),
\end{gathered}
$$

or

$$
\begin{aligned}
H_{\alpha_{1} \ldots \alpha_{n+1}}(\vec{r})= & \frac{(n+1) n}{2}-\frac{n}{2} \sum_{i}\left(U_{\alpha_{1} \ldots \alpha_{i-1} \alpha_{i+1} \ldots \alpha_{n+1}}(\vec{r})+U_{\alpha_{1} \ldots \alpha_{i-1} \alpha_{i+1} \ldots \alpha_{n+1}}\left(\vec{r}-\vec{e}_{\alpha_{i}}\right)\right) \\
+ & \frac{1}{4} \sum_{i<k}\left(U_{\alpha_{1} \ldots \alpha_{i-1} \alpha_{i+1} \ldots \alpha_{n+1}}(\vec{r})+U_{\alpha_{1} \ldots \alpha_{i-1} \alpha_{i+1} \ldots \alpha_{n+1}}\left(\vec{r}-\vec{e}_{\alpha_{i}}\right)\right) \\
& \left(U_{\alpha_{1} \ldots \alpha_{k-1} \alpha_{k+1} \ldots \alpha_{n+1}}(\vec{r})+U_{\alpha_{1} \ldots \alpha_{k-1} \alpha_{k+1} \ldots \alpha_{n+1}}\left(\vec{r}-\vec{e}_{\alpha_{k}}\right)\right) .
\end{aligned}
$$

Again as before $(7 \mathrm{a}, \mathrm{b})$ the hyperplaquette variables $U$ are not independent. Because the hyperedge $\Omega_{\alpha_{1} \ldots \alpha_{n+1}}(\vec{r})$ belongs totally to an even number, $2(n+1)$, of hyperplaquettes $\Omega_{\alpha_{1} \ldots \alpha_{k-1} \alpha_{k+1} \ldots \alpha_{n+1}}(\vec{r})$ and $\Omega_{\alpha_{1} \ldots \alpha_{k-1} \alpha_{k+1} \ldots \alpha_{n}}\left(\vec{r}-\vec{e}_{\alpha_{k}}\right)$, the product of the corresponding hyperplaquette variables is always one. Thus one has constraints

$$
\prod_{k=1}^{n+1} U_{\alpha_{1} \ldots \alpha_{k-1} \alpha_{k+1} \ldots \alpha_{n+1}}(\vec{r}) U_{\alpha_{1} \ldots \alpha_{k-1} \alpha_{k+1} \ldots \alpha_{n+1}}\left(\vec{r}-\vec{e}_{\alpha_{k}}\right)=1 .
$$

To resolve constraints (7c), let us attach an Ising spin $\sigma$ in the center of each $d-n+1$ dimensional hypercube $\Omega_{\alpha_{1} \ldots \alpha_{n-1}}(\vec{r})$. Then there exist spin configurations $\sigma$, so that the hyperplaquette variables $U$ obey 国

$$
U_{\alpha_{1} \ldots \alpha_{n}}(\vec{r})=\prod_{k=1}^{n} \sigma_{\alpha_{1} \ldots \alpha_{k-1} \alpha_{k+1} \ldots \alpha_{n}}(\vec{r}) \sigma_{\alpha_{1} \ldots \alpha_{k-1} \alpha_{k+1} \ldots \alpha_{n}}\left(\vec{r}-\vec{e}_{\alpha_{k}}\right)
$$

The $\sigma$ 's are defined by this equations up to global and /or local gauge transformations [4]. Equations (5b) together with (21) and (9c) completely solve the problem.

Now let us consider special cases. For $n=1$ this yields the competing interaction for Ising spins

$$
\begin{aligned}
H_{\alpha \beta}(\vec{r}) & =1-\frac{1}{2}\left(U_{\alpha}(\vec{r})+U_{\alpha}\left(\vec{r}-\vec{e}_{\beta}\right)+U_{\beta}(\vec{r})+U_{\beta}\left(\vec{r}-\vec{e}_{\alpha}\right)\right) \\
& +\frac{1}{2}\left(U_{\alpha}(\vec{r})+U_{\alpha}\left(\vec{r}-\vec{e}_{\beta}\right)\left(U_{\beta}(\vec{r})+U_{\beta}\left(\vec{r}-\vec{e}_{\alpha}\right)\right),\right.
\end{aligned}
$$

which yields

$$
H_{\alpha \beta}(\vec{r})=
$$




$$
\begin{gathered}
1-\frac{1}{2}\left(\sigma(\vec{r}) \sigma\left(\vec{r}-\vec{e}_{\alpha}\right)+\sigma\left(\vec{r}-\vec{e}_{\beta}\right) \sigma\left(\vec{r}-\vec{e}_{\alpha}-\vec{e}_{\beta}\right)+\sigma(\vec{r}) \sigma\left(\vec{r}-\vec{e}_{\beta}\right)+\sigma\left(\vec{r}-\vec{e}_{\alpha}\right) \sigma\left(\vec{r}-\vec{e}_{\alpha}-\vec{e}_{\beta}\right)\right) \\
+\frac{1}{2}\left(\sigma\left(\vec{r}-\vec{e}_{\alpha}\right) \sigma\left(\vec{r}-\vec{e}_{\beta}\right)+\sigma(\vec{r}) \sigma\left(\vec{r}-\vec{e}_{\alpha}-\vec{e}_{\beta}\right)\right) .
\end{gathered}
$$

Thus the Hamiltonian reads

$$
\begin{gathered}
H=\sum_{\vec{r}}\left\{\frac{d(d-1)}{2}-(d-1) \sum_{\alpha} \sigma(\vec{r}) \sigma\left(\vec{r}-\vec{e}_{\alpha}\right)\right. \\
\left.+\frac{1}{2} \sum_{\alpha<\beta}\left(\sigma\left(\vec{r}-\vec{e}_{\alpha}\right) \sigma\left(\vec{r}-\vec{e}_{\beta}\right)+\sigma(\vec{r}) \sigma\left(\vec{r}-\vec{e}_{\alpha}-\vec{e}_{\beta}\right)\right)\right\} .
\end{gathered}
$$

and again this Hamiltonian represents a magnetic system with competing coupling constants adjusted to

$$
J_{\text {ferromagnet }}=2(d-1) J_{\text {antiferromagnet }} \text {. }
$$

For $n=2$ one obtains the gauge spin system

$$
\begin{gathered}
H_{\alpha \beta \gamma}(\vec{r})=3-\left(U_{\alpha \beta}(\vec{r})+U_{\alpha \beta}\left(\vec{r}-\vec{e}_{\gamma}\right)\right. \\
\left.+U_{\alpha \gamma}(\vec{r})+U_{\alpha \gamma}\left(\vec{r}-\vec{e}_{\beta}\right)+U_{\beta \gamma}(\vec{r})+U_{\beta \gamma}\left(\vec{r}-\vec{e}_{\alpha}\right)\right) \\
+\frac{1}{4}\left(U_{\alpha \beta}(\vec{r})+U_{\alpha \beta}\left(\vec{r}-\vec{e}_{\gamma}\right)\right)\left(U_{\alpha \gamma}(\vec{r})+U_{\alpha \gamma}\left(\vec{r}-\vec{e}_{\beta}\right)\right) \\
+\frac{1}{4}\left(U_{\alpha \beta}(\vec{r})+U_{\alpha \beta}\left(\vec{r}-\vec{e}_{\gamma}\right)\right)\left(U_{\beta \gamma}(\vec{r})+U_{\beta \gamma}\left(\vec{r}-\vec{e}_{\alpha}\right)\right) \\
+\frac{1}{4}\left(U_{\alpha \gamma}(\vec{r})+U_{\alpha \gamma}\left(\vec{r}-\vec{e}_{\beta}\right)\right)\left(U_{\beta \gamma}(\vec{r})+U_{\beta \gamma}\left(\vec{r}-\vec{e}_{\alpha}\right)\right)
\end{gathered}
$$

and

$$
\begin{gathered}
H=\sum_{\vec{r}}\left\{\frac{d(d-1)(d-2)}{2}-2(d-2) \sum_{\alpha<\beta} U_{\alpha \beta}(\vec{r})\right. \\
\left.+\frac{1}{4} \sum_{\alpha, \beta<\gamma}\left(U_{\alpha \beta}(\vec{r})+U_{\alpha \beta}\left(\vec{r}-\vec{e}_{\gamma}\right)\right)\left(U_{\alpha \gamma}(\vec{r})+U_{\alpha \gamma}\left(\vec{r}-\vec{e}_{\beta}\right)\right)\right\},
\end{gathered}
$$

where

$$
U_{\alpha \beta}(\vec{r})=\sigma_{\alpha}(\vec{r}) \sigma_{\alpha}\left(\vec{r}-\vec{e}_{\beta}\right) \sigma_{\beta}(\vec{r}) \sigma_{\beta}\left(\vec{r}-\vec{e}_{\alpha}\right)
$$

and

$$
J_{\text {plaquettes }}=8(d-2) J_{\text {rt plaquettes }} \text {. }
$$

Thus one has a "ferromagnetic" interaction on each elementary plaquette and an "antiferromagnetic" one on double-plaquettes which form a right angle. 


\section{Comments}

Let us consider the case of one-dimensional hypersurfaces $M_{1}, d-n=1$, that is random walks on the lattice, with the property that the straight "motion" does not cost an energy, any right angle turns cost $\Theta(\pi / 2)$ and for self intersections the number of pairs of links meeting under a right angle times $\Theta(\pi / 2)$ has to be paid. As it is easy to see from our general result, when we change the dimension of the target space $R^{d}$, then we also should change the index $n$. In the two-dimensional case we have $n=d-1=1$ and the spin Hamiltonian of the type (11),(24). This special case is of particular interest, because it can be mapped [5, 6] to the eightvertex model [7] with the weights

$$
\begin{gathered}
\epsilon_{1}=\epsilon_{3}=\epsilon_{4}=0, \\
\epsilon_{5}=\epsilon_{6}=\epsilon_{7}=\epsilon_{8}=\Theta(\pi / 2) .
\end{gathered}
$$

This is not the exactly solvable zero-field Baxter model, since $\epsilon_{1} \neq \epsilon_{2}$. When $d=3$, for this type of scale invariant random walks we should consider gauge spin system with $n=2$ and so on.

The models with $n=1$ are BNNNI (biaxial next-nearest- neighbor Ising) models which are relatives of the ANNNI (anisotropic next-nearest-neighbor Ising) models

[8, 9, 10, 11, 12, 13] with specially adjusted coupling constants, the restrictions of which are dictated by the string geometry. It seems that the model with $d=2, n=1$ does not show a phase-transition [8, 14]. For $d=3$ this is a random surface model $M_{2}$, for $d=4$ a membrane model $M_{3}$. In four and higher dimensions the random surface models $M_{2}$ are again gauge models.

\section{Acknowledgements}

One of the authors (G.K.S.) is grateful to E. Floratos, R. Flume, H. Fritsche, N. Papanicolaou, E. Paschos and T. Tomaras for fruitful discussions and to W. Greiner for useful comments and hospitality in Frankfurt University.

This work was supported in part by the Alexander von Humboldt Foundation. 


\section{References}

[1] R.V. Ambartzumian, G.K. Savvidy, K.G. Savvidy and G.S. Sukiasian. Phys. Lett. B275 (1992) 99

[2] G.K. Savvidy and K.G. Savvidy. String fine tuning. Preprint UFTP 302/1992, hep-th 9208041,Int. J. Mod. Phys. A (1993)

[3] G.K. Savvidy, K.G. Savvidy. Gonihedric String and Asymptotic Freedom. Preprint UFTP 320/1992, hep-th 9301001

[4] F.J. Wegner, J. Math. Phys.12 (1971) 2259

[5] L.P. Kadanoff and F.J. Wegner, Phys. Rev. B4 (1971) 3989

[6] R.Y. Wu, Phys.Rev. B4 (1971) 2312

[7] R.J.Baxter, Exactly solved models in statistical mechanics. Academic Press, London 1982

[8] W. Selke, Physics Reports 170 (1988) 213

[9] C. Domb, Adv. Phys. 9 (1960) 149

[10] R.J. Elliott, Phys. Rev.124 (1961) 346

[11] P. Bak and J. von Boehm, Phys. Rev. Lett. 42 (1980) 122

[12] M.E. Fisher and W.Selke, Phys. Rev. Lett. 44(1980) 1502

[13] E.I. Dinaburg and Ya.G. Sinai, Comm.Math.Phys. 98 (1985) 119

[14] D.P. Landau, K. Binder, Phys. Rev. B 31 (1985) 5946 